\title{
Universal transverse momentum dependent soft function at NNLO
}

\author{
Miguel G. Echevarria, ${ }^{1,2, *}$ Ignazio Scimemi, ${ }^{3, \dagger}$ and Alexey Vladimirov ${ }^{4, \$}$ \\ ${ }^{1}$ Nikhef Theory Group, Science Park 105, 1098XG Amsterdam, The Netherlands \\ ${ }^{2}$ Department of Physics and Astronomy, VU University Amsterdam, De Boelelaan 1081, \\ NL-1081 HV Amsterdam, The Netherlands \\ ${ }^{3}$ Departamento de Física Teórica II, Facultad de Ciencias Físicas, \\ Universidad Complutense de Madrid, 28040 Madrid, Spain \\ ${ }^{4}$ Institut für Theoretische Physik, Universität Regensburg, D-93040 Regensburg, Germany
}

(Received 30 November 2015; published 2 March 2016)

\begin{abstract}
All (un)polarized transverse momentum dependent functions (TMDs), both distribution and fragmentation functions, are defined with the same universal soft function, which cancels spurious rapidity divergences within an individual TMD and renders them well-defined hadronic quantities. Moreover, it is independent of the kinematics, whether it is Drell-Yan, deep inelastic scattering, or $e^{+} e^{-} \rightarrow 2$ hadrons. In this paper, we provide this soft function at next-to-next-to-leading order (NNLO), necessary for the calculation of all TMDs at the same order, and to perform the resummation of large logarithms at next-tonext-to-next-to-leading-logarithmic accuracy. From the results we obtain the $D$ function at NNLO, which governs the evolution of all TMDs. This work represents the first independent and direct calculation of this quantity. Given the all-order relation through a Casimir scaling between the soft function relevant for gluon TMDs and the one for quark TMDs, we also obtain the first at NNLO. The used regularization method to deal with the rapidity divergences is discussed as well.
\end{abstract}

DOI: $10.1103 /$ PhysRevD.93.054004

\section{INTRODUCTION}

In the recent works that establish the factorization theorems for the transverse momentum spectra in Drell-Yan (DY), vector or Higgs boson production, semiinclusive deep inelastic scattering (SIDIS), and $e^{+} e^{-} \rightarrow 2$ hadrons [1-4], the soft function (SF) plays a central role. The main building blocks within these factorization theorems are the transverse momentum dependent functions (TMDs), which encode the relevant nonperturbative physics. And the SF enters these TMDs in a particular way, in order to cancel spurious rapidity divergences and make them well-defined hadronic quantities.

The $\mathrm{SF}$ is defined as the vacuum expectation value of a product of lightlike Wilson lines evaluated at a spacelike distance. It is an utterly singular object which is undefined without a proper regularization method and cannot be considered as a probability amplitude in the usual quantum field theory sense. Given the way it is introduced and used, the SF is similar to the renormalization constants of the quantum field theory ( $Z$ factors), which are undefined without regularization, and has individual physical meaning: while the renormalization constants subtract the ultraviolet (UV) divergences of diagrams, the SF subtracts the rapidity divergences, the most specific divergences of TMD factorization.

\footnotetext{
*m.g.echevarria@nikhef.nl

ignazios@fis.ucm.es

*vladimirov.aleksey@gmail.com
}

When calculating the SF, one faces divergences of different types, and only some of them can be controlled by dimensional regularization (with $d \neq 4$ ). Therefore, it is important to understand the origin of these divergences.

The main attention is paid to the rapidity divergences, as they represent the most crucial point in the calculation of the SF. These divergences arise when loop momentum, denoted by $k$, is boosted in one light-cone direction (given by the vectors $n_{ \pm}$with $n_{+}^{2}=n_{-}^{2}=0$ and $n_{+} n_{-}=2$ ), say, $k^{+} \rightarrow \infty$ and $k^{-} \rightarrow 0$, keeping $k^{+} k^{-}$fixed. The important point is that lightlike Wilson lines, and therefore the SF, are invariant under the coordinate rescaling in their own lightlike directions. This invariance leads to an ambiguity in the definition of rapidity divergences. Indeed, the boost $k^{+} \rightarrow a k^{+}, k^{-} \rightarrow k^{-} / a$ (with $a$ an arbitrary number) leaves the SF invariant, while in the limit $a \rightarrow \infty$ reproduces the rapidity divergent configuration. Therefore, without a regularization which breaks the boost invariance, the SF cannot be explicitly calculated. In this sense, rapidity divergences cannot be regularized by any type of dimensional-like regularization.

There are many ways to break boost invariance and make the rapidity divergences manifest. One way is to tilt the Wilson lines from the light cone, such that $n_{ \pm}^{2}>0$ and infinitesimal. This procedure implies the use of different soft factors to deal with the self-energies of Wilson lines and avoid double counting; see e.g. $[1,5,6]$. In these works, one combines the unevaluated integrands of collinear and soft matrix elements together to form integrals free from rapidity divergences. Implementing these combinations at 
two loops can be demanding. From the calculational point of view, we find it much more economical to have a sufficient regularization for the TMDs, such that every diagram gives a finite result. It also grants a higher universality of the obtained expressions, since many diagrams and even parts of calculations appear in different objects and can be reused. The SF is a good example of such universality, as once it is evaluated at next-to-next-toleading order (NNLO), it can be used to calculate all leading-twist TMDs at the same order. In this respect, the formulation of the factorization theorem of Ref. [2] is more suitable for a NNLO evaluation, and we use it in the definitions of this paper.

Given the importance that the SF has per se for the establishment of the factorization theorem, we provide its explicit NNLO calculation in the present work. The SF is defined as

$$
\begin{aligned}
S\left(\boldsymbol{k}_{s \perp}\right)= & \int \frac{d^{2} \boldsymbol{b}_{\perp}}{(2 \pi)^{2}} e^{i \boldsymbol{b}_{\perp} \cdot \boldsymbol{k}_{s \perp}} \frac{\operatorname{Tr}_{c}}{N_{c}} \\
& \times\left\langle 0\left|\left[S_{n}^{T \dagger} \tilde{S}_{\bar{n}}^{T}\right]\left(0^{+}, 0^{-}, \boldsymbol{b}_{\perp}\right)\left[\tilde{S}_{\bar{n}}^{T \dagger} S_{n}^{T}\right](0)\right| 0\right\rangle,
\end{aligned}
$$

where $S_{n}$ and $\tilde{S}_{\bar{n}}$ stand for soft Wilson lines along the light-cone directions $n$ and $\bar{n}$, respectively $\left(n^{2}=\bar{n}^{2}=0\right.$, $n \cdot \bar{n}=2$ ). The superscript $T$ on Wilson lines in Eq. (1) implies subsidiary transverse gauge links from the light-cone infinities to transverse infinity (see more details in Refs. [7-9]). These links guarantee gauge invariance and are necessary for calculations in singular gauges. The present calculation has been performed in the Feynman gauge, where the contribution of transverse links vanishes.

The establishment of the factorization theorem to all orders in the perturbation theory relies on particular properties of the SF with respect to the rapidity regulator. Essentially, the logarithm of the SF is at most linear in the logarithms generated by the rapidity divergences [using the $\delta$ regularization, to be introduced in Eq. (5) in the next section]. This guaranties that the SF can be factorized in two pieces [3], and in turn it allows one to define the individual TMDs. With the $\delta$ regularization, this important relation reads

$$
\tilde{S}\left(\mathbf{L}_{\mu}, \mathbf{L}_{\sqrt{\delta^{+} \delta^{-}}}\right)=\tilde{S}^{\frac{1}{2}}\left(\mathbf{L}_{\mu}, \mathbf{L}_{\delta^{+} / \nu}\right) \tilde{S}^{\frac{1}{2}}\left(\mathbf{L}_{\mu}, \mathbf{L}_{\nu \delta^{-}}\right),
$$

where tildes mark quantities calculated in coordinate space, $\nu$ is an arbitrary and positive real number that transforms as $p^{+}$under boosts, and we introduce the convenient notation

$$
\mathbf{L}_{X} \equiv \ln \left(X^{2} \boldsymbol{b}^{2} e^{2 \gamma_{E}} / 4\right) .
$$

Note that the relation in Eq. (2) is exact; i.e. it is valid to all orders in the perturbation theory, as well as to all orders in the $\epsilon$ expansion. To the best of our knowledge, there is no general proof of this statement. Consequently, one of the goals of our calculation is to establish the relation in Eq. (2) at NNLO and thus verify the TMD factorization theorem at the same order.

The fact that one has a unique SF for different processes (with a different composition of initial and/or final states) is a direct consequence of the linear dependence on the logarithms of the rapidity divergences. Moreover, one can extract the TMD evolution function $D$ [3] from the SF (see Sec. IV). In this paper, we obtain it explicitly at NNLO and establish the Casimir scaling between the $D$ function relevant for quark TMDs and for gluon TMDs, which is valid to all orders in the perturbation theory. The realization of the cancellation of rapidity divergences at NNLO within one single TMD has been explicitly checked for the first time in Ref. [10] for the case of the unpolarized nonsinglet fragmentation functions, and its generalization for the whole set of unpolarized TMDs is in preparation [11].

In the literature, we have found that an early calculation of the SF was done in Ref. [12] using just dimensional regularization, which is not appropriate to deal with rapidity divergences. Before the development of the current TMD formalism, the universality of soft radiation was discussed in Ref. [13] at the one-loop level. In the context of TMDs, the first soft function appeared in the work of Ji, $\mathrm{Ma}$, and Yuan $[14,15]$. In that work, it was argued that the SF in coordinate space should depend just on the transverse coordinate. However, the regularization of the SF was done using tilted Wilson lines, and properly defining the TMDs by using that regulator requires the combination of several types of soft functions, as later discussed in Ref. [1]. Moreover, Ji, Ma, and Yuan proposed the subtraction of the whole SF in the definition of the TMDs, which does not provide a complete cancellation of all rapidity divergences within one TMD. Collins argued in [1] that in order to properly define one single TMD it is necessary to combine three soft functions in coordinate space and still use tilted Wilson lines. The computation of (three) SF using Wilson lines off the light cone turns out to be not practical at higher orders in the perturbation theory. The calculation of the SF on the light cone has been attempted by several authors, and it is not free from difficulties [2,3,16-18]. In Ref. [18], for instance, the integrals of the soft function are all scaleless to all orders in the perturbation theory, so that the regulator that they propose is not suitable for establishing the TMD formalism; however, it appears to be very efficient for the calculation of the total cross section. The calculation of the DY cross section done at two loops in Refs. $[19,20]$ in this sense is equivalent to the QCD calculation performed in Ref. [21]. In Ref. [17], the rapidity divergences are regularized by explicitly breaking Lorentz invariance through the introduction of a rapidity regulator. A twoloop calculation of the SF using this regulator has not been performed yet to our knowledge. The regulator proposed here allows the calculation of a single TMD and has been used for the complete calculation of the nonsinglet 
unpolarized TMD fragmentation function in Ref. [10], using the TMD factorization theorem in the EchevarriaIdilbi-Scimemi formalism [2,3].

The explicit form of Wilson lines together with the regularization method that we have used is detailed in Sec. II. The result of the calculation of the SF at NNLO and the related discussion appears in Sec. III. In Sec. IV, we discuss the properties of the SF, and, in particular, we argue that the universal evolution function $D$ for all TMDs can be extracted solely from the SF, for both gluons and quarks. Finally, we conclude in Sec. V. The technical details of our calculations are given in the Appendixes.

\section{DEFINITIONS AND REGULARIZATION}

The SF is written more easily in coordinate space, where it takes the form

$\tilde{S}\left(\boldsymbol{b}_{T}\right)=\frac{\operatorname{Tr}_{c}}{N_{c}}\left\langle 0\left|T\left[S_{n}^{T \dagger} \tilde{S}_{\bar{n}}^{T}\right]\left(0^{+}, 0^{-}, \boldsymbol{b}_{T}\right) \bar{T}\left[\tilde{S}_{\bar{n}}^{T \dagger} S_{n}^{T}\right](0)\right| 0\right\rangle$,

where we explicitly denote the ordering of operators. The Wilson lines are defined as

$$
\begin{aligned}
S_{n}^{T} & =T_{n(\bar{n})} S_{n}, \\
\tilde{S}_{\bar{n}}^{T} & =\tilde{T}_{n(\bar{n})} \tilde{S}_{\bar{n}}, \\
S_{n}(x) & =P \exp \left[i g \int_{-\infty}^{0} d s n \cdot A(x+s n)\right], \\
T_{n}(x) & =P \exp \left[i g \int_{-\infty}^{0} d \tau \vec{l}_{\perp} \cdot \vec{A}_{\perp}\left(\infty^{+}, 0^{-}, \vec{x}_{\perp}+\vec{l}_{\perp} \tau\right)\right], \\
T_{\bar{n}}(x) & =P \exp \left[i g \int_{-\infty}^{0} d \tau \vec{l}_{\perp} \cdot \vec{A}_{\perp}\left(0^{+}, \infty^{-}, \vec{x}_{\perp}+\vec{l}_{\perp} \tau\right)\right], \\
\tilde{S}_{\bar{n}}(x) & =P \exp \left[-i g \int_{0}^{\infty} d s \bar{n} \cdot A(x+\bar{n} s)\right], \\
\tilde{T}_{n}(x) & =P \exp \left[-i g \int_{0}^{\infty} d \tau \vec{l}_{\perp} \cdot \vec{A}_{\perp}\left(\infty^{+}, 0^{-}, \vec{x}_{\perp}+\vec{l}_{\perp} \tau\right)\right], \\
\tilde{T}_{\bar{n}}(x) & =P \exp \left[-i g \int_{0}^{\infty} d \tau \vec{l}_{\perp} \cdot \vec{A}_{\perp}\left(0^{+}, \infty^{-}, \vec{x}_{\perp}+\vec{l}_{\perp} \tau\right)\right],
\end{aligned}
$$

and transverse gauge links $T_{n(\bar{n})}$ appear for the gauge choice $n \cdot A=0$ (or $\bar{n} \cdot A=0$ ), while the rest of the Wilson lines appearing in Eq. (1) are obtained by exchanging $n \leftrightarrow \bar{n}$ and path-ordering $P$ with anti-path-ordering $\bar{P}$. We notice that these definitions apply for SIDIS kinematics [4], but, as we will show below, the soft function turns out to be universal.

The choice of the IR and rapidity regularization scheme is one of the key ingredients in the calculation of TMDs. In our work, we choose to regularize the rapidity divergences with the $\delta$ regularization, that has been already used for the same purpose by many authors (see e.g. [2,4,22,23]). In its original definition, $\delta$ regularization consists in setting the $i 0$ prescription of eikonal propagators finite. However, this definition appears to be inefficient for high-loop computations. In particular, it breaks the non-Abelian exponentiation property of Wilson lines, which is crucial for TMD factorization to hold.

Thus, in order to provide a more efficient computation we regularize the UV and IR-soft (mass) divergences using standard dimensional regularization with $D=4-2 \varepsilon$. Then for rapidity divergences we define a $\delta$-regularization scheme at the operator level, which consists in modifying the definition of Wilson lines as

$$
\begin{aligned}
\tilde{S}_{\bar{n}}(0) & =P \exp \left[-i g \int_{0}^{\infty} d \sigma A_{+}(\sigma \bar{n})\right] \\
& \rightarrow P \exp \left[-i g \int_{0}^{\infty} d \sigma A_{+}(\sigma \bar{n}) e^{-\delta^{+} \sigma}\right], \\
S_{n}(0) & =P \exp \left[i g \int_{-\infty}^{0} d \sigma A_{-}(\sigma n)\right] \\
& \rightarrow P \exp \left[i g \int_{-\infty}^{0} d \sigma A_{-}(\sigma n) e^{+\delta^{-} \sigma}\right],
\end{aligned}
$$

where $\delta^{ \pm} \rightarrow 0^{+}$. The modified operator supplies the nonAbelian exponentiation property. To be explicit, at the level of Feynman diagrams in momentum space, the modified expressions for the eikonal propagators are written as (e.g. absorbing gluons by the Wilson line $\left[\infty^{+}, 0\right]$ )

$$
\begin{aligned}
& \frac{1}{\left(k_{1}^{+}-i 0\right)\left(k_{2}^{+}-i 0\right) \ldots\left(k_{n}^{+}-i 0\right)} \\
& \rightarrow \frac{1}{\left(k_{1}^{+}-i \delta^{+}\right)\left(k_{2}^{+}-2 i \delta^{+}\right) \ldots\left(k_{n}^{+}-n i \delta^{+}\right)},
\end{aligned}
$$

where the gluons are ordered from infinity to zero (i.e. $k_{n}$ is the gluon closest to zero). As a consequence of the rescaling invariance of the Wilson lines (that is now explicitly broken by the parameters $\delta^{ \pm}$), the expressions for diagrams in the SF depend on the single variable $2 \delta^{+} \delta^{-} /(n \bar{n})=\delta^{+} \delta^{-}$.

The ordering of poles in the eikonal propagators Eq. (6) is crucial for perturbative exponentiation with the usual properties, such as the non-Abelian exponentiation theorem on color factors [24,25] or logarithmical counting [26]. The origin of these properties is the color ordering of the gluon fields along the path of the Wilson line, that results in a specific nested-commutator structure for exponentiated operators [27,28]. The regularization in Eq. (5) definitely preserves the ordering, while naive $\delta$ regularization (with $i 0$ directly replaced by $i \delta$ ) corresponds to some involved operator with mixed color ordering and violates exponentiation properties. On the level of Feynman graphs, the problems of naive $\delta$ regularization can be already seen at NNLO, where terms with color factors $C_{F}^{2}$ arise in the 
exponent. Concluding, within the modified $\delta$ regularization, only diagrams with non-Abelian color prefactor (web diagrams) arise in the exponent. Thus, the result is conveniently presented in the form

$$
\tilde{S}\left(b_{T}\right)=\exp \left[a_{s} C_{F}\left(S^{[1]}+a_{s} S^{[2]}+\cdots\right)\right],
$$

where $a_{s}=g^{2} /(4 \pi)^{2}$ is the strong coupling and $C_{F}$ is the Casimir of the fundamental representation of the gauge group $\left[C_{F}=\left(N_{c}^{2}-1\right) / N_{c}\right.$ for $\left.S U\left(N_{c}\right)\right]$.

The Wilson lines in the modified $\delta$ regularization in Eq. (5) do not have the gauge properties of the original Wilson lines. However, the gauge transformation properties are restored in the limit $\delta \rightarrow 0^{+}$. Therefore, only the calculation in this limit is appropriate. In the calculation of Feynman diagrams, this implies that terms linear (and higher powers) in $\delta$ should be neglected. In this case, the gauge invariance of the final result is guaranteed.

Important to mention is that a naive implementation of the $\delta$ regulator can cause the appearance of spurious terms that would pretend to violate gauge invariance. This situation takes place in some diagrams at the two-loop level, and in Appendix B we discuss how these terms should be properly handled and thus gauge invariance recovered.

Now, once we have introduced both the object we want to calculate and the regularization scheme we implement, we comment on the general structure of the result. For a generic two-loop diagram, dimensional analysis gives

$$
\text { Diagram }=\mu^{4 \epsilon}\left(A_{0} \boldsymbol{\delta}^{-2 \epsilon}+A_{1} \boldsymbol{\delta}^{-\epsilon} \boldsymbol{B}^{\epsilon}+A_{2} \boldsymbol{B}^{2 \epsilon}\right)+\mathcal{O}(\delta),
$$

where

$$
\boldsymbol{\delta}= \pm \delta^{+} \delta^{-}, \quad \boldsymbol{B}=\frac{b_{T}^{2}}{4} .
$$

The sign of $\boldsymbol{\delta}$ depends on the kinematics of the SF [as it can be deduced from Eq. (5)]: it is plus for Drell-Yan and $e^{+} e^{-}$annihilation processes and minus for SIDIS. When all diagrams are summed up, the final result is linear in $\ln |\boldsymbol{\delta}|$ at all orders in $\epsilon$ expansion. The linearity in $\ln |\boldsymbol{\delta}|$ ensures the splitting of the SF in two pieces as in Eq. (2) and, consequently, the cancellation of rapidity divergences between the SF and the collinear matrix element inside any given well-defined TMD (and, therefore, the validity of the factorization theorem as well). Moreover, the modulus $|\boldsymbol{\delta}|$ makes this cancellation independent of the kinematics, being a manifestation of the universality of the SF.

The logarithms generated by rapidity divergences are partially included in the coefficient $A_{2}$, which is a polynomial of $\ln (\boldsymbol{\delta} \boldsymbol{B})$. The coefficients $A_{0}$ and $A_{1}$ are just functions of $\epsilon$. Both $A_{0}$ and $A_{1}$ terms cancel exactly in the sum of diagrams. The cancellation of the sum of the $A_{0}$ terms comes from the trivial fact that the integrated SF $(\boldsymbol{B}=0)$ is null at all positive orders in the perturbative expansion. Thus, it would not be necessary to calculate any completely virtual diagram, since they contribute only to $A_{0}$. However, we calculate them and explicitly check their exact cancellation. The cancellation of the $A_{1}$ term has a similar origin. In fact, the integrated SF should also be recovered by taking the particular limit $\boldsymbol{B} \rightarrow 0$ with $\boldsymbol{B} / \boldsymbol{\delta}$ fixed. Then, the only way to recover the integrated SF is to set $A_{1}=0$ in the sum of all diagrams. Thus, the final result does not contain any fractional powers of $\delta$. In this way, the only $\delta$ dependence in the final result is logarithms $\ln (\boldsymbol{\delta} \boldsymbol{B})$ appearing in $A_{2}$. Notice that this cancellation is also expected from the formulation of the relevant factorization theorems, where the SF is implied to be linear in the logarithms generated by rapidity divergences to all orders in $\epsilon$ and, hence, cannot contain $\delta^{-\epsilon}$ terms. The rapidity divergences appear in $A_{2}$ only as $\ln ^{n}(\boldsymbol{\delta} \boldsymbol{B})$ with $n=1,2$, but $\ln ^{2}(\boldsymbol{\delta} \boldsymbol{B})$ cancels in the sum of all diagrams.

At higher loops, we expect a similar structure of divergences in each diagram: at order $n$, the general expression for a diagram reads $\mu^{2 n \epsilon} \sum_{k=0}^{n} A_{k} \boldsymbol{\delta}^{-(n-k) \epsilon} \boldsymbol{B}^{k \epsilon}$. Similar considerations show that in the sum of all diagrams only the term $\mu^{2 n \epsilon} A_{n} \boldsymbol{B}^{n \epsilon}$ survives. Diagram by diagram, the coefficient $A_{n}$ can contain powers of $\ln (\boldsymbol{\delta} \boldsymbol{B})$ up to the order of $n$, while in the sum of all diagrams only terms linear in $\ln |\boldsymbol{\delta}|$ remain.

Finally, we observe that, in order to smoothly recover the integrated SF limit with $\boldsymbol{B} \rightarrow 0$, for all terms of the type $\boldsymbol{B}^{n \epsilon}$ we need $\epsilon>0$. That is also supported by the loop integrals. In this way, all $\epsilon$ divergences of the final result are of UV origin.

We recall that there are two sources for the UV $\epsilon$ poles of the final result. One is the standard surface UV divergence of loop integrals, i.e. when $k^{2} \rightarrow \infty$. Another one is the rapidity divergence $k^{ \pm} \rightarrow \infty$ at fixed $k^{2}$. We stress that the rapidity divergences appear as both UV and IR divergences. From the UV side the rapidity divergence is regularized by dimensional regularization, while from the IR side it is regularized by the $\delta$ regularization. In the final result, the rapidity divergences appear as both $\ln (\delta)$ and $\epsilon$ poles.

Let us summarize the singularity structure of the diagrams contributing to the SF at the two-loop level:

(i) The poles in $\epsilon$ can have at most a power $\epsilon^{-4}$. Because of the renormalization theorem for Wilson lines $[29,30]$, the $\epsilon^{-4}$ poles cancel in the sum of all diagrams. Therefore, the final result has at most $\epsilon^{-3}$ poles.

(ii) In each diagram, there are logarithms of $\delta$ arising from the expansion of $\boldsymbol{\delta}^{-\epsilon}$ [corresponding to the terms $A_{0}$ and $A_{1}$ in Eq. (8)]. Up to the order of $\mathcal{O}\left(\epsilon^{0}\right)$, one can have at most $\ln ^{4} \boldsymbol{\delta}$ contribution. These divergences cancel in the combination of all diagrams at all orders in $\epsilon$.

(iii) Finally, there are logarithms $\ln (\boldsymbol{\delta} \boldsymbol{B})$ (only inside the coefficient $A_{2}$ ), and in each diagram one can have at most $\ln ^{2}(\boldsymbol{\delta} \boldsymbol{B})$. The logarithms squared cancel in the sum of diagrams. The remaining single logarithms are the only dependence on $\delta$ in the final result. 


\section{SOFT FUNCTION AT NLO AND NNLO}

The evaluation of the diagrams for the SF is a delicate process. One should pay a lot of attention to the analytical properties of loop integrals, since any mistake in the $i 0$ prescription gives rise to phase factors that, multiplied by $\epsilon^{-4}$, result in a wrong combination of singularities. Mistakes of such a type are very difficult to trace, and they would result into a breakdown of the TMD factorization. Thus, we make an evaluation of all diagrams in the form shown in Eq. (8), without any expansion in $\epsilon$, in order to trace all factors and singularities explicitly. Then, combining diagrams by sectors we check that terms with $\boldsymbol{\delta}^{-\epsilon}$ completely cancel with each other. This provides a very strong check of our loop integrals and for the final result.

\section{A. Soft function at NLO}

The NLO diagrams are shown in Fig. 1, and the result reads

$$
\begin{aligned}
\mathrm{S}_{A}^{[1]}= & -\frac{2 g^{2}}{(4 \pi)^{\frac{d}{2}}} \mu^{2 \epsilon} \boldsymbol{\delta}^{-\epsilon} \Gamma^{2}(\epsilon) \Gamma(1-\epsilon), \\
\mathrm{S}_{B}^{[1]}= & \frac{2 g^{2}}{(4 \pi)^{\frac{d}{2}}} \mu^{2 \epsilon}\left[\boldsymbol{\delta}^{-\epsilon} \Gamma^{2}(\epsilon) \Gamma(1-\epsilon)\right. \\
& \left.-\boldsymbol{B}^{\epsilon} \Gamma(-\epsilon)\left(L_{+}-\psi(-\epsilon)-\gamma_{E}\right)\right],
\end{aligned}
$$

where

$$
L_{ \pm}=\ln \left( \pm \frac{\boldsymbol{B}|\boldsymbol{\delta}|}{e^{-2 \gamma_{E}}}\right)
$$

and the suffixes $A$ and $B$ refer to diagrams in Fig. 1. In these expressions, the general structure discussed in the previous section is transparent.

Adding the mirror diagram of diagram $B$ and the complex conjugated diagram to diagram $A$, we finally obtain the complete NLO result for the SF:

$$
S^{[1]}=-4 \mu^{2 \epsilon} \boldsymbol{B}^{\epsilon} \Gamma(-\epsilon)\left(L_{0}-\psi(-\epsilon)-\gamma_{E}\right),
$$
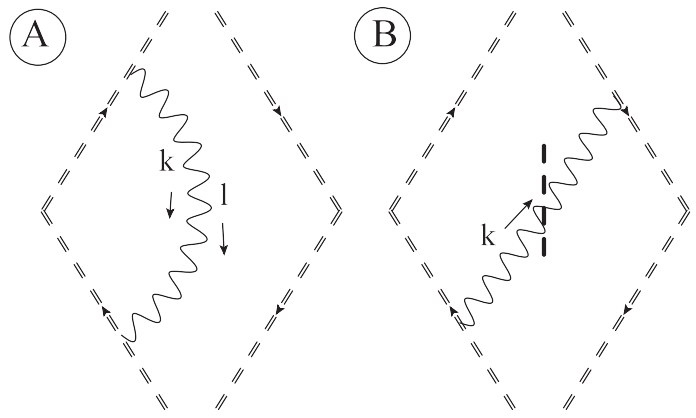

FIG. 1. One-loop diagrams contributing to the soft factor. The conjugated and mirror diagrams should be added. where $\psi$ stands for the digamma function and

$$
L_{0}=\ln \left(\frac{\boldsymbol{B}|\boldsymbol{\delta}|}{e^{-2 \gamma_{E}}}\right) \text {. }
$$

The result in Eq. (11) fulfills all basic properties of the SF. Indeed, it is linear in $L_{0}$ at all orders in $\epsilon$, and thus it depends solely on $|\boldsymbol{\delta}|$.

Evaluation of the one-loop diagrams clearly illustrates the structure of SF divergences discussed in the previous section. So, to evaluate diagram $A$ we keep $\epsilon>0$, that allows us to interpret the resulting double epsilon pole as an UV pole. Simultaneously, the double UV pole appearing in diagram $A$ has an entwined structure. This double pole is collected from two sources: the surface UV singularity (that comes from the integral over $k_{T}$ ) and the rapidity divergence (that come from the integral over $k^{ \pm}$). Meanwhile, diagram $B$ is not $\epsilon$-divergent and can be evaluated with both positive and negative $\epsilon$. To keep the uniform scheme, we assume $\epsilon>0$, which also supports the smooth limit $\boldsymbol{B} \rightarrow 0$. Relying on the positivity of $\epsilon$, we interpret the remaining $\epsilon$ poles in the sum of diagrams in Eq. (11) as UV poles. The same conclusion has been made in Ref. [31], where extra gluon-mass regulator was used for more detailed control of IR divergences. A similar structure, but with extra complications from interference of IR and UV regions, holds as well for the NNLO result.

Expanding Eq. (11) in $\epsilon$, we obtain [we use the standard $\overline{\mathrm{MS}}$ prescription $\left.\mu^{2} \rightarrow \mu^{2} e^{\gamma_{E}} /(4 \pi)\right]$

$S^{[1]}=-\frac{4}{\epsilon^{2}}+2 \mathbf{L}_{\mu}^{2}-\frac{2 d^{(1,1)}}{C_{F}}\left(\frac{1}{\epsilon}+\mathbf{L}_{\mu}\right) \mathbf{l}_{\delta}+\frac{\pi^{2}}{3}+\mathcal{O}(\epsilon)$,

where $\mathbf{l}_{\delta}=\ln \left(\mu^{2} /|\boldsymbol{\delta}|\right)$ and $d^{(1,1)}=2 C_{F}=\Gamma_{0} / 2$. This expression agrees with previous calculations [2,4,22,32]. Notice that for the NNLO calculation we need $S^{[1]}$ up to the order of $\epsilon^{3}$ which can be derived directly from Eq. (11). The coefficient $d^{(1,1)}$ refers to the quark TMD evolution $D$ function at one loop. We elaborate on this at all orders in Sec. IV.

\section{B. Soft function at NNLO}

The two-loop diagrams are shown in Fig. 2. In this figure, they have been grouped in virtual-virtual (VV, diagrams $A-D$ ), virtual-real (VR, diagrams $E-I$ ), and real-real (RR, diagrams $K-P$ ). Below, we discuss the main results and features of the calculation, while the technical details of the evaluation of these diagrams can be found in Appendix A. We recall that $\mathrm{VV}$ diagrams contribute only to the $A_{0}$ terms in Eq. (8), so they cancel completely in the sum of all diagrams.

Besides ordering the diagrams in VV, VR, and RR, the two-loop web diagrams can be naturally split into four categories: 
(i) diagrams with vacuum-polarization subdiagrams $(C$, $D, H, I, O, P)$;

(ii) multigluon exchange webs (MGEW) $(A, E, K, L)$;

(iii) diagrams with a three-gluon vertex $(B, F, G, M$, $N)$; and

(iv) counterterms that come from the renormalization of the Wilson lines.

These subclasses represent sectors of QCD interactions with different properties. Therefore, it is convenient to present the expression for the SF in the form

$$
S^{[2]}=S_{\mathrm{pol}}^{[2]}-\frac{C_{A}}{2} S_{\mathrm{MGEW}}^{[2]}-\frac{C_{A}}{2} S_{3 g}^{[2]}+S_{c t}^{[2]} .
$$

The mixed singularities proportional to $\boldsymbol{\delta}^{-\epsilon}$ cancel inside every class of diagrams. This happens because these classes represent separate parts of QCD interactions, while the cancellation is dictated by the structure of the operator. The confirmation of the cancellation inside every class of diagrams is a very strong check of the calculation. Another check is the cancellation of the leading $\epsilon^{-4}$ pole. Because of the QCD Ward identities, the cancellation takes place between MGEW and three-gluon interaction diagrams.

The result of the contribution of the diagrams with a vacuum polarization subdiagram is

$$
\begin{aligned}
S_{\mathrm{pol}}^{[2]}= & 8 \mu^{4 \epsilon} \boldsymbol{B}^{2 \epsilon}\left[C_{A}(5-3 \epsilon)-4 T_{R} N_{f}(1-\epsilon)\right] \\
& \times \frac{\Gamma(-2 \epsilon) \Gamma(-\epsilon) \Gamma(2-\epsilon)}{\Gamma(4-2 \epsilon)}\left(L_{0}-\psi(-2 \epsilon)-\gamma_{E}\right) \\
& -8 \mu^{2 \epsilon} \boldsymbol{B}^{\epsilon}\left(\frac{2}{3} T_{R} N_{f}-\frac{5}{6} C_{A}\right) \frac{\Gamma(-\epsilon)}{\epsilon} \\
& \times\left(L_{0}-\psi(-\epsilon)-\gamma_{E}\right) .
\end{aligned}
$$

The term proportional to $\boldsymbol{B}^{2 \epsilon}$ in Eq. (15) comes entirely from diagram $O$. The part of diagram $O$ proportional to $\boldsymbol{\delta}^{\epsilon}$ cancels with similar contributions coming from other diagrams (see explicit expressions in Appendix A). Diagrams $H$ and $I$ are zero in dimensional regularization; however, their UV counterterms are not. The term proportional to $\boldsymbol{B}^{\epsilon}$ in Eq. (15) is the contribution of the UV renormalization of diagram $H$, and it is proportional to the one-loop diagram $B$, Eq. (10), multiplied by gluon propagator renormalization constant $Z_{3, \overline{\mathrm{MS}}}^{1 / 2}$ :

$$
Z_{3, \overline{\mathrm{MS}}}=1-\frac{a_{s}}{3 \epsilon}\left(4 T_{R} N_{f}-5 C_{A}\right)+\mathcal{O}\left(a_{s}^{2}\right) .
$$

Notice that $S_{\text {pol }}^{[2]}$ is linear in $L_{0}$.

The contribution of MGEW diagrams collected together is

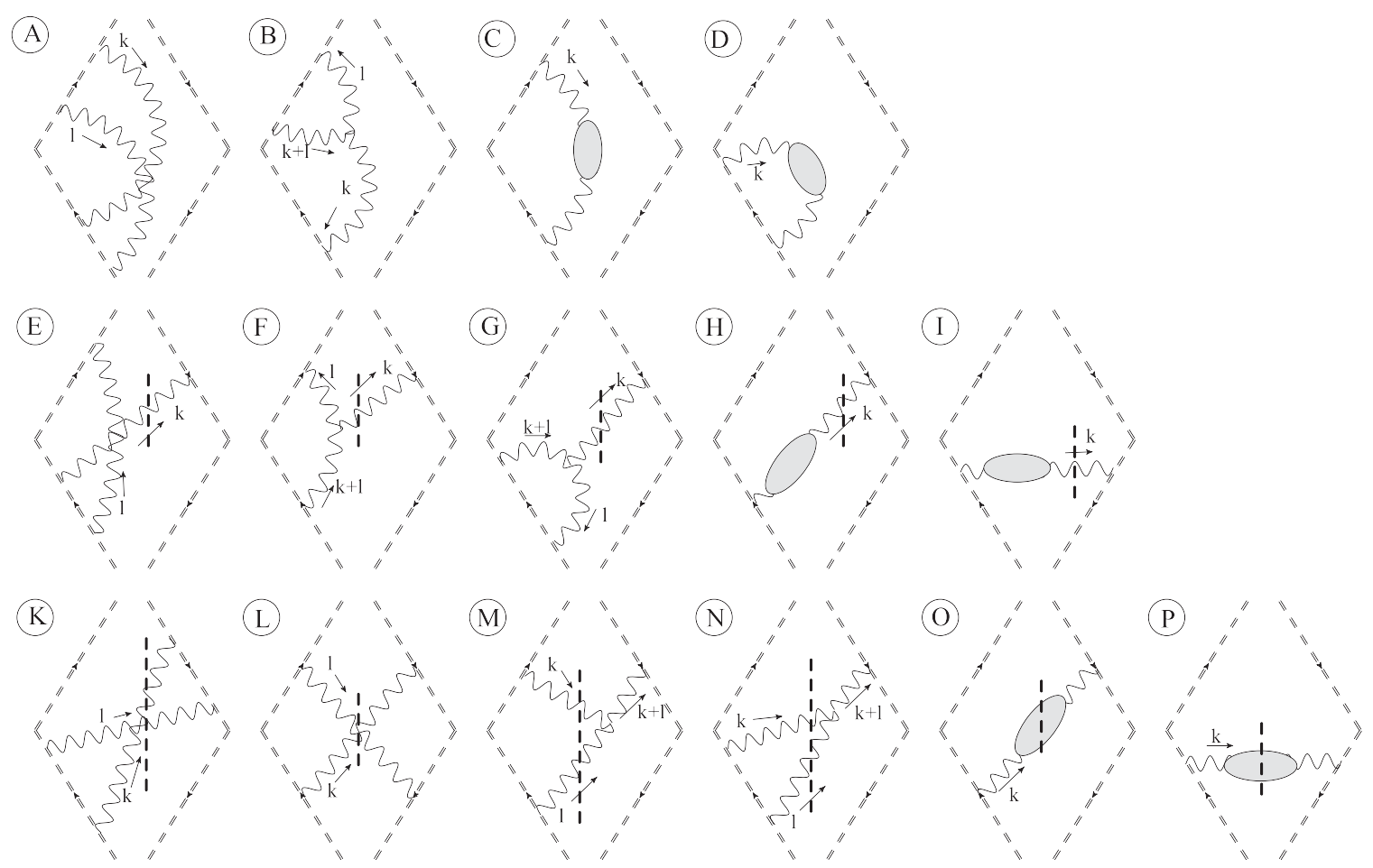

FIG. 2. Two-loop diagrams contributing to the soft factor grouped in virtual-virtual (VV, diagrams $A-D$ ), virtual-real (VR, diagrams $E-I)$, and real-real (RR, diagrams $K-P$ ). The conjugated and mirror diagrams should be added. The gray blob denotes the vacuumpolarization subdiagram. 


$$
\begin{aligned}
S_{\mathrm{MGEW}}^{[2]} & =4 \mu^{4 \epsilon} \boldsymbol{B}^{2 \epsilon}\left\{\Gamma^{2}(-\epsilon)\left(L_{0}-\psi(-\epsilon)-\gamma_{E}\right)^{2}+4 \Gamma(-2 \epsilon) \Gamma(-\epsilon) \Gamma(\epsilon)\left(\psi\left(\frac{1-\epsilon}{2}\right)-\Gamma(-\epsilon) \Gamma(1+\epsilon)\right)+4 Q(\epsilon)\right. \\
& \left.+\Gamma^{2}(-\epsilon)\left[\left(2 L_{0}+2 \gamma_{E}+8 \ln 2-\psi(-2 \epsilon)-3 \psi(-\epsilon)\right)(\psi(-\epsilon)-\psi(-2 \epsilon))+3 \psi^{\prime}(-2 \epsilon)-2 \psi^{\prime}(-\epsilon)-\frac{5 \pi^{2}}{6}\right]\right\},
\end{aligned}
$$

where the function $Q(\epsilon)=\mathcal{O}\left(\epsilon^{0}\right)$ and it is given in Eq. (A7).

The result for the three-gluon interaction diagrams is

$$
\begin{aligned}
S_{3 g}^{[2]}= & -4 \mu^{4 \epsilon} \boldsymbol{B}^{2 \epsilon} \Gamma^{2}(-\epsilon)\left\{\left(L_{0}-\psi(-\epsilon)-\gamma_{E}\right)^{2}\right. \\
& +2\left[\left(L_{0}-\psi(-2 \epsilon)-\gamma_{E}\right)\left(\frac{1}{1-2 \epsilon}+\psi(-2 \epsilon)-\psi(1-\epsilon)+\psi(1+\epsilon)+\gamma_{E}\right)+\frac{\ln 2}{1-2 \epsilon}-\frac{\pi^{2}}{6}\right. \\
& +\psi^{\prime}(-\epsilon)+\frac{1}{2} \psi^{\prime}(1+\epsilon)-\frac{3}{2} \psi^{\prime}(-2 \epsilon)-\frac{1}{2}\left(\psi(-\epsilon)+\gamma_{E}\right)\left(2 \psi(-2 \epsilon)-3 \psi(-\epsilon)-\gamma_{E}\right) \\
& \left.\left.+\frac{1}{2}\left(\psi(-2 \epsilon)+\psi(1+\epsilon)+2 \gamma_{E}\right)(3 \psi(-2 \epsilon)-4 \psi(-\epsilon)+\psi(1+\epsilon))+\frac{\psi(-2 \epsilon)-\psi(-\epsilon)}{\epsilon}-\frac{1}{2 \epsilon^{2}}\right]\right\} .
\end{aligned}
$$

The logarithms $L_{0}$ appear quadratically and linearly in both Eqs. (17) and (18). However, in their sum the $L_{0}^{2}$ terms cancel with each other, leaving a linear dependence on $L_{0}$. It is worth mentioning that this cancellation leaves a trace in the form of terms proportional to $\pi^{2}$. It happens because, individually, diagrams depend on $L_{ \pm}$and their complex conjugates. In the final result, the complex phases cancel, and Eqs. (17) and (18) are naturally expressed via $L_{0}$.

Finally, we should add the renormalization factor for Wilson lines, that comes from the renormalization of both the coupling constant and the gluon fields. It is proportional to the one-loop diagrams multiplied by $Z_{g \overline{\mathrm{MS}}} Z_{3 \overline{\mathrm{MS}}}^{1 / 2} . Z_{3 \overline{\mathrm{MS}}}$ is given in Eq. (16), and

$$
Z_{g \overline{\mathrm{MS}}}=1-\frac{a_{s}}{6 \epsilon}\left(11 C_{A}-4 T_{R} N_{f}\right)+\mathcal{O}\left(a_{s}^{2}\right)
$$

which add up to

$$
S_{c t}^{[2]}=8 a_{s}^{2} C_{F} C_{A} \frac{\Gamma(-\epsilon)}{\epsilon} \mu^{2 \epsilon} \boldsymbol{B}^{\epsilon}\left(L_{0}-\gamma_{E}-\psi(-\epsilon)\right) .
$$

Combining all pieces together as in Eq. (14), we obtain the final expression for the SF. Here we explicitly write the result expanded in $\epsilon$ :

$$
\begin{aligned}
S^{[2]}= & \frac{d^{(2,2)}}{C_{F}}\left(\frac{3}{\epsilon^{3}}+\frac{2 \mathbf{l}_{\delta}}{\epsilon^{2}}+\frac{\pi^{2}}{6 \epsilon}+\frac{4}{3} \mathbf{L}_{\mu}^{3}-2 \mathbf{L}_{\mu}^{2} \mathbf{l}_{\delta}+\frac{2 \pi^{2}}{3} \mathbf{L}_{\mu}+\frac{14}{3} \zeta_{3}\right)-\frac{d^{(2,1)}}{C_{F}}\left(\frac{1}{2 \epsilon^{2}}+\frac{\mathbf{l}_{\delta}}{\epsilon}-\mathbf{L}_{\mu}^{2}+2 \mathbf{L}_{\mu} \mathbf{l}_{\delta}-\frac{\pi^{2}}{4}\right) \\
& -\frac{d^{(2,0)}}{C_{F}}\left(\frac{1}{\epsilon}+2 \mathbf{l}_{\delta}\right)+C_{A}\left(\frac{\pi^{2}}{3}+4 \ln 2\right)\left(\frac{1}{\epsilon^{2}}+\frac{2 \mathbf{L}_{\mu}}{\epsilon}+2 \mathbf{L}_{\mu}^{2}+\frac{\pi^{2}}{6}\right)+C_{A}\left(8 \ln 2-9 \zeta_{3}\right)\left(\frac{1}{\epsilon}+2 \mathbf{L}_{\mu}\right) \\
& +\frac{656}{81} T_{R} N_{f}+C_{A}\left(-\frac{2428}{81}+16 \ln 2-\frac{7 \pi^{4}}{18}-28 \ln 2 \zeta_{3}+\frac{4}{3} \pi^{2} \ln ^{2} 2-\frac{4}{3} \ln ^{4} 2-32 \operatorname{Li}_{4}\left(\frac{1}{2}\right)\right)+\mathcal{O}(\epsilon) .
\end{aligned}
$$

The coefficients $d^{(n, k)}$ are the coefficients of the $D$ function at two loops which governs the TMD evolution kernel (see [33], which uses a slightly different notation):

$$
\begin{gathered}
d^{(2,2)}=\frac{\Gamma^{(0)} \beta_{0}}{4}=C_{F}\left(\frac{11}{3} C_{A}-\frac{4}{3} T_{R} N_{f}\right), \quad d^{(2,1)}=\frac{\Gamma^{(1)}}{2}=2 C_{F}\left(\left(\frac{67}{9}-\frac{\pi^{2}}{3}\right) C_{A}-\frac{20}{9} T_{R} N_{f}\right) \\
d^{(2,0)}=C_{F}\left(\left(\frac{404}{27}-14 \zeta_{3}\right) C_{A}-\frac{112}{27} T_{R} N_{f}\right) .
\end{gathered}
$$

We discuss the relation between the SF and the $D$ function more extensively in the next section.

The final result in Eq. (21) contains terms with $\ln 2$, which are atypical in loop calculations. This is a feature of the $\delta$ regulator. However, these terms cancel within an object free from rapidity divergences (see the result for the TMD fragmentation function obtained in Ref. [10]). 


\section{EXTRACTION OF THE EVOLUTION FACTOR $D$ FROM THE SF}

The evolution factor $D$ for quark TMDs can be extracted directly from the SF. This feature is fundamental to prove the universality of the TMD evolution. In fact, because of the universality (or process independence) of the SF, the function $D$ is also universal and appears in the evolution of all (un)polarized TMDs, both distribution and fragmentation functions.

To extract the evolution factor $D$, we recall the definition of a generic TMD in the light cone + direction

$$
\begin{aligned}
T_{+}\left(z, b_{T} ; \zeta_{+}, \mu\right)= & Z_{2}^{-1} Z_{T}\left(\frac{\zeta_{+}}{\mu^{2}}\right) T_{+}^{\mathrm{unsub}}\left(z, b_{T} ; \mu, \frac{\delta^{+}}{p^{+}}\right) \\
& \times \tilde{S}^{-1 / 2}\left(b_{T} ; \mu, \delta^{+} / \nu\right),
\end{aligned}
$$

where $Z_{2}$ is the quark wave-function renormalization constant, $Z_{T}$ is the TMD operator renormalization constant, and $\tilde{S}$ is evaluated in the previous section. The variable $\zeta_{+}=\left(p^{+} / \nu\right)^{2}$ is the rapidity scale that arises from the freedom in the splitting procedure of the SF (see e.g. $[10,33]$ for more details), with $\nu$ an arbitrary and positive real number that transforms as $p^{+}$under boosts [cf. Eq. (2)].

The dependence on $\delta^{+}$is enclosed in the SF and the unsubtracted TMD, while the rapidity scale variation is dictated by the TMD renormalization factor $Z_{T}$ and the SF.

The TMD evolution equation with respect to the rapidity parameter defines the evolution factor $D$. It reads

$$
\frac{d}{d \ln \zeta_{+}} T_{+}=-D T_{+}
$$

Only the SF and the renormalization constant $Z_{T}$ are $\zeta$ dependent. Therefore, we rewrite

$$
\frac{d \ln Z_{T}\left(\frac{\zeta_{+}}{\mu^{2}}\right)}{d \ln \zeta_{+}}-\frac{1}{2} \frac{d \ln \tilde{S}\left(\delta^{+} \frac{\sqrt{\zeta_{+}}}{p^{+}}\right)}{d \ln \zeta_{+}}=-D,
$$

where we drop unnecessary arguments of functions. Finally, using the relation in Eq. (2), we obtain

$$
D=\frac{1}{2} \frac{d \ln \tilde{S}}{d \mathbf{l}_{\delta}}-\frac{d \ln Z_{T}}{d \ln \zeta_{+}} .
$$

Evaluating the derivative of the SF, we obtain a result that contains $\epsilon$ poles. However, these $\epsilon$ poles are removed by the renormalization constant $Z_{T}$ in Eq. (26), so the function $D$ is finite. It is important that renormalization constant $Z_{T}$ contains only $\epsilon$ poles and does not contain finite $\ln (\zeta)$ dependence. Therefore, we can extract the function $D$ by differentiating the SF and neglecting all $\epsilon$ poles. The perturbative result for the $D$ function can be written as

$$
D=\sum_{n} \sum_{k=0}^{n} a_{s}^{n} \boldsymbol{L}_{\mu}^{k} d^{(n, k)},
$$

the NLO coefficients are $d^{(1,0)}=0, d^{(1,1)}$ is given after Eq. (13), while NNLO coefficients $d^{(2, i)}$ are given in Eq. (22). The calculation of the SF performed in this work so represents the first direct calculation of the $D$ function at NNLO and agrees with a previous derivation of the result $[2,18]$.

We conclude noting that a remarkable property of nonAbelian exponentiation is that the $S U(3)$ generators in the Wilson lines enter as a global multiplier of the exponent; see Eq. (4). In this way, the difference between soft functions that use different $S U(3)$ representations is a multiplicative factor at all orders in the perturbation theory. This implies that the $D$ functions for gluons, $D_{g}$, and quarks, $D$, are related by

$$
\frac{D}{C_{F}}=\frac{D_{g}}{C_{A}}
$$

at all orders in the perturbation theory.

\section{CONCLUSIONS}

The definition and calculation of the soft function is crucial in TMD factorization theorems. In fact, one has just one soft function for all quark TMDs (and one for all gluon TMDs), which enters their definition in a specific way to make them well-defined hadronic quantities. This relies on the single logarithmic dependence of the soft function in the logarithm generated by rapidity divergences, which allows its splitting in rapidity space. In this work, we have reported on the NNLO calculation of this soft function, using the $\delta$ regulator for rapidity divergences, and explicitly checked for the first time its splitting in rapidity space at this nontrivial order. Moreover, we have performed the calculation to all orders in dimensional regularization, which allows the extraction of the general structure of the soft function to all orders.

From the result, we have obtained the $D$ function at NNLO, which represents the first independent and direct calculation of this universal quantity, relevant for the evolution of TMDs. Furthermore, given that the relation between the quark and gluon soft functions is just a Casimir scaling at all orders in the perturbation theory, we have also obtained the NNLO soft function relevant for gluon TMDs, as well as its $D_{g}$ term at the same order.

Finally, since the soft function is universal and enters the definition of all (un)polarized TMD distribution and fragmentation functions, this calculation represents an important ingredient in order to obtain the relevant perturbative coefficients to resum large logarithms for any TMD. A first application of this soft function which represents also a strong check of the present calculation is reported in Ref. [10]. 


\section{ACKNOWLEDGMENTS}

A. V. thanks Victor Svensson for helpful discussions. M. G. E. is supported by the "Stichting voor Fundamenteel Onderzoek der Materie" (FOM), which is financially supported by the "Nederlandse Organisatie voor Wetenschappelijk Onderzoek" (NWO). I. S. is supported by the Spanish MECD Grants No. FPA2011-27853-CO202 and No. FPA2014-53375-C2-2-P.

\section{APPENDIX A: EXPRESSIONS FOR DIAGRAMS AND LOOP INTEGRALS}

In this Appendix, we present the expressions for individual diagrams, which are shown in Figs. 1 and 2. We use the notation $S_{X}^{[n]}$ for the contribution of diagram $X$ to $S^{[n]}$, defined in Eq. (7).

The calculation of the loop integrals was performed in the following way. First, we integrate over one of the light-cone components, using either the $\delta$ function for real gluons or the Cauchy theorem for virtual components. Second, we integrate over transverse components. Here for real gluons we make the Mellin-Barnes expansion, such that the integrand contains only powers of the transverse components, such that the Fourier integral can be easily performed. Third, we integrate over the residual light-cone component. Finally, we are left with an integral of the Mellin-Barnes type, that can be straightforwardly evaluated by closing the integration contour in the half-plane with suppressed $\boldsymbol{\delta}$. Typically, one needs to consider only the residues in the vicinity of zero, since we need only the leading terms in $\delta \rightarrow 0$. However, for some integrals (e.g. $I_{A}^{\prime \prime}$ ), powers of $\delta$ can be compensated between the Mellin-Barnes integrals, and the complete sum of residues should be considered.

The one-loop diagrams are

$$
\begin{gathered}
S_{A}^{[1]}=-\mu^{2 \epsilon} K_{1}^{(0)}, \\
S_{B}^{[1]}=-\mu^{2 \epsilon} K_{1}{ }^{\prime} .
\end{gathered}
$$

The two-loop diagrams with two virtual gluons are

$$
\begin{aligned}
S_{A}^{[2]}= & -\frac{C_{A}}{2} \mu^{4 \epsilon} I_{A}, \\
S_{B}^{[2]}= & -\frac{C_{A}}{2} \mu^{4 \epsilon}\left(2 I_{C 1}+I_{C 2}\right), \\
S_{C}^{[2]}= & -4 \Gamma(\epsilon) \frac{\Gamma(1-\epsilon) \Gamma(3-\epsilon)}{\Gamma(5-2 \epsilon)} \\
& \times\left[C_{A}(5-3 \epsilon)-4 T_{R} N_{f}(1-\epsilon)\right] \mu^{4 \epsilon}\left(K_{1}^{(\epsilon)}+\ldots\right),
\end{aligned}
$$

where the dots denote a term which is zero if the $\delta$ regulator is implemented properly (see the explanation in Appendix B). Diagram $D$ is zero (see also Appendix B). The two-loop diagrams with a single real gluon are

$$
\begin{aligned}
S_{E}^{[2]} & =-\frac{C_{A}}{2} \mu^{4 \epsilon} I_{A}{ }^{\prime}, \\
S_{F}^{[2]} & =\frac{C_{A}}{2} \mu^{4 \epsilon}\left(I_{C 3}^{\prime}-I_{C 4}^{\prime}\right), \\
S_{G, H, I}^{[2]} & =0,
\end{aligned}
$$

where the latter are zero are due to the absence of a scale within the virtual loop. Finally, the diagrams with two real gluons are

$$
\begin{aligned}
S_{K}^{[2]}= & -\frac{C_{A}}{2} \mu^{4 \epsilon} I_{A}^{\prime \prime}, \\
S_{L}^{[2]}= & -\frac{C_{A}}{2} \mu^{4 \epsilon}\left|K_{1}^{\prime}\right|^{2}, \\
S_{M}^{[2]}= & \frac{C_{A}}{2} \mu^{4 \epsilon}\left(I_{C 1}^{\prime \prime}+I_{C 2}^{\prime \prime}\right), \\
S_{N}^{[2]}= & \frac{C_{A}}{2} \mu^{4 \epsilon}\left(I_{C 3}^{\prime \prime}-2 I_{C 4}^{\prime \prime}\right), \\
S_{O}^{[2]}= & -2\left(C_{A}(5-3 \epsilon)-4 T_{R} N_{f}(1-\epsilon)\right) \\
& \times \frac{\Gamma(2-\epsilon)}{\Gamma(4-2 \epsilon)} \mu^{4 \epsilon}\left(K_{1}^{\prime \prime}+\ldots\right),
\end{aligned}
$$

where the dots denote a term which is zero if the $\delta$ regulator is implemented properly (see the explanation in Appendix B). Diagram $P$ is zero for the same reason (see also Appendix B).

The expressions for one-loop-like integrals are

$$
\begin{aligned}
K_{1}^{(a)} & =\int \frac{d^{d} k}{(2 \pi)^{d}} \frac{-i}{\left(k^{+}+i \delta^{+}\right)\left(k^{-}+i \delta^{-}\right)\left(-k^{2}-i 0\right)^{1+a}} \\
& =2 \boldsymbol{\delta}^{-\epsilon-a} \frac{\Gamma^{2}(a+\epsilon) \Gamma(1-a-\epsilon)}{\Gamma(1+a)}, \\
K_{1}^{\prime} & =\int \frac{d^{d} k}{(2 \pi)^{d}} \frac{(-2 \pi) \theta\left(k^{+}\right) \delta\left(k^{2}\right) e^{i(k b)_{T}}}{\left(k^{+}+i \delta^{+}\right)\left(k^{-}+i \delta^{-}\right)} \\
& =-2\left[\boldsymbol{\delta}^{-\epsilon} \Phi_{\epsilon}-\boldsymbol{B}^{\epsilon} \Psi_{\epsilon}\right], \\
K_{1}^{\prime \prime} & =\int \frac{d^{d} k}{(2 \pi)^{d}} \frac{(2 \pi) \theta\left(k^{+}\right) \theta\left(k^{2}\right) e^{i(k b)_{T}}\left(k^{2}\right)^{1-\epsilon}}{\left(k^{+}+i \delta^{+}\right)\left(k^{-}+i \delta^{-}\right)\left(k^{2}+i 0\right)\left(k^{2}-i 0\right)} \\
& =2 \Gamma(-\epsilon)\left[\delta^{-2 \epsilon} \Phi_{2 \epsilon}-\boldsymbol{B}^{2 \epsilon} \Psi_{2 \epsilon}\right],
\end{aligned}
$$

where we use the shorthand notation

$$
\begin{aligned}
& \Phi_{\epsilon}=\Gamma^{2}(\epsilon) \Gamma(1-\epsilon), \\
& \Psi_{\epsilon}=\Gamma(-\epsilon)\left(L_{+}-\psi(-\epsilon)-\gamma_{E}\right) .
\end{aligned}
$$

The expressions for the MGEW integrals are 


$$
\begin{aligned}
I_{A}= & \int \frac{d^{d} k d^{d} l}{(2 \pi)^{2 d}} \frac{-1}{\left(k^{+}-i \delta^{+}\right)\left(k^{+}+l^{+}-2 i \delta^{+}\right)\left(l^{-}-i \delta^{-}\right)\left(l^{-}+k^{-}-2 i \delta^{-}\right)\left(k^{2}+i 0\right)\left(l^{2}+i 0\right)}=\frac{1}{4}\left(K_{1}^{(0)}\right)^{2}, \\
I_{A}^{\prime}= & \int \frac{d^{d} k d^{d} l}{(2 \pi)^{2 d}} \frac{(-2 \pi i) \theta\left(k^{+}\right) \delta\left(k^{2}\right) e^{i(k b)_{T}}}{\left(l^{+}+i \delta^{+}\right)\left(l^{-}+i \delta^{-}\right)\left(l^{-}+k^{-}+2 i \delta^{-}\right)\left(k^{+}+i \delta^{+}\right)\left(l^{2}+i 0\right)} \\
= & K_{1}^{(0)} K_{1}^{\prime}+4 \Phi_{\epsilon}\left(\frac{\boldsymbol{\delta}^{-2 \epsilon}}{2} \Phi_{\epsilon}+\boldsymbol{B}^{\epsilon} \boldsymbol{\delta}^{-\epsilon} F_{\epsilon}+\boldsymbol{B}^{2 \epsilon} \Gamma(-2 \epsilon) \Gamma(-\epsilon)\right), \\
I_{A}^{\prime \prime}= & \int \frac{d^{d} k d^{d} l}{(2 \pi)^{2 d}} \frac{(-2 \pi)^{2} \theta\left(k^{+}\right) e^{i(k b)_{T}} \delta\left(k^{2}\right) \theta\left(l^{+}\right) e^{i(l b)_{T}} \delta\left(l^{2}\right)}{\left(l^{+}+i \delta^{+}\right)\left(k^{+}+l^{+}+2 i \delta^{+}\right)\left(l^{-}+i \delta^{-}\right)\left(k^{-}+l^{-}+2 i \delta^{-}\right)}=\frac{\left(K_{1}^{\prime}\right)^{2}}{2}-\boldsymbol{\delta}^{-2 \epsilon} \Phi_{\epsilon}^{2}-8 \boldsymbol{B}^{\epsilon} \boldsymbol{\delta}^{-\epsilon} \Phi_{\epsilon} F_{\epsilon} \\
& -2 \boldsymbol{B}^{2 \epsilon} \Psi_{\epsilon}^{2}+4 \boldsymbol{B}^{2 \epsilon} \Phi_{\epsilon}(\psi(-\epsilon)-\psi(-2 \epsilon))+8 \boldsymbol{B}^{2 \epsilon}\left(\Gamma(-2 \epsilon) \Gamma(-\epsilon) \Gamma(\epsilon) \psi\left(\frac{1-\epsilon}{2}\right)+Q(\epsilon)\right) \\
& +2 \boldsymbol{B}^{2 \epsilon} \Gamma^{2}(-\epsilon)\left[\frac{\pi^{2}}{6}+3 \psi^{\prime}(-2 \epsilon)-2 \psi^{\prime}(-\epsilon)+\left(\psi(-2 \epsilon)-\psi(-\epsilon)+4 \gamma_{E}+8 \ln 2\right)(\psi(-\epsilon)-\psi(-2 \epsilon))\right],
\end{aligned}
$$

where

$$
\begin{aligned}
F_{\epsilon} & =2^{1-\epsilon} \frac{\Gamma(-\epsilon)}{\epsilon}{ }_{2} F_{1}(1,1,1+\epsilon ;-1) \\
Q(\epsilon) & =\sum_{k=1}^{\infty} \frac{(-1)^{k}}{k !} \Gamma(k-\epsilon)\left(\Gamma(k) \Gamma(-k-\epsilon) \psi\left(\frac{1+k}{2}\right)+\Gamma(k-2 \epsilon) \Gamma(\epsilon-k) \psi\left(\frac{1+k-\epsilon}{2}\right)\right) .
\end{aligned}
$$

The integrals involving the three-gluon vertex are

$$
\begin{aligned}
I_{C 1}= & \int \frac{d^{d} k d^{d} l}{(2 \pi)^{2 d}} \frac{1}{\left(k^{+}+i \delta^{+}\right)\left(k^{-}+2 i \delta^{-}\right)\left(k^{2}+i 0\right)\left(l^{2}+i 0\right)\left[(k+l)^{2}+i 0\right]}=2^{-2 \epsilon} \Gamma(\epsilon) \frac{\Gamma^{2}(1-\epsilon)}{\Gamma(2-2 \epsilon)} K_{1}^{(\epsilon)}, \\
I_{C 2}= & \int \frac{d^{d} k d^{d} l}{(2 \pi)^{2 d}} \frac{1}{\left(k^{+}+i \delta^{+}\right)\left(l^{-}-i \delta^{-}\right)\left(k^{2}+i 0\right)\left(l^{2}+i 0\right)\left[(k+l)^{2}+i 0\right]}=2 \delta^{-2 \epsilon} \Phi_{2 \epsilon} \Gamma(\epsilon) \Gamma(-\epsilon), \\
I_{C 3}^{\prime}= & \int \frac{d^{d} k d^{d} l}{(2 \pi)^{2 d}} \frac{(-2 \pi i) \theta\left(k^{+}\right) \delta\left(k^{2}\right) e^{i(k b)_{T}}}{\left(l^{+}+i \delta^{+}\right)\left(k^{-}+l^{-}+i \delta^{-}\right)\left(l^{2}+i 0\right)\left((k+l)^{2}+i 0\right)}=-I_{C 1}^{\prime \prime}-2 I_{C 2}-4 \boldsymbol{B}^{\epsilon} \boldsymbol{\delta}^{-\epsilon} \Gamma^{2}(\epsilon) \Gamma^{2}(-\epsilon) \\
& +\boldsymbol{B}^{\epsilon} \Gamma^{2}(-\epsilon)\left[\left(L_{+}+\psi(-2 \epsilon)+\psi(1+\epsilon)-2 \psi(-\epsilon)\right)^{2}+2 \psi^{\prime}(-\epsilon)+\psi^{\prime}(1+\epsilon)-3 \psi^{\prime}(-2 \epsilon)+\frac{2 \pi^{2}}{3}\right], \\
I_{C 4}^{\prime}= & \int \frac{d^{d} k d^{d} l}{(2 \pi)^{2 d}} \frac{(-2 \pi i) \theta\left(k^{+}\right) \delta\left(k^{2}\right) e^{i(k b)_{T}}}{\left(k^{+}+i \delta^{+}\right)\left(k^{-}+l^{-}+i \delta^{-}\right)\left(l^{2}+i 0\right)\left((k+l)^{2}+i 0\right)}=0, \\
I_{C 1}^{\prime \prime}= & \int \frac{d^{d} k d^{d} l}{(2 \pi)^{2 d}} \frac{(-2 \pi)^{2} \theta\left(k^{+}\right) \delta\left(k^{2}\right) e^{i(k b)_{T}} \theta\left(l^{+}\right) \delta\left(l^{2}\right) e^{i(l b)_{T}}}{\left(k^{+}-i \delta^{+}\right)\left(l^{-}+i \delta^{-}\right)\left((k+l)^{2}-i 0\right)} \\
= & 2(-\delta)^{-2 \epsilon} \Phi_{2 \epsilon} \Gamma(\epsilon) \Gamma(-\epsilon)+\boldsymbol{B}^{2 \epsilon} \Gamma^{2}(-\epsilon)\left[\left(L_{0}+\psi(-2 \epsilon)-\psi(-\epsilon)\right)^{2}-3 \psi^{\prime}(-2 \epsilon)+\psi \psi^{\prime}(-\epsilon)+\frac{2 \pi^{2}}{3}\right], \\
I_{C 2}^{\prime \prime}= & I_{C 3}^{\prime \prime}=\int \frac{d^{d} k d^{d} l}{(2 \pi)^{2 d}} \frac{(-2 \pi)^{2} \theta\left(k^{+}\right) \delta\left(k^{2}\right) e^{i(k b)_{T}} \theta\left(l^{+}\right) \delta\left(l^{2}\right) e^{i(l b)_{T}}}{\left(k^{+}+l^{+}+i \delta^{+}\right)\left(l^{-}+i \delta^{-}\right)\left((k+l)^{2}-i 0\right)} \\
= & 2 \delta^{-2 \epsilon} \Phi_{2 \epsilon} \Gamma(\epsilon) \Gamma(-\epsilon)+2 \boldsymbol{B}^{\epsilon} \boldsymbol{\delta}^{-\epsilon} \Gamma^{2}(\epsilon) \Gamma^{2}(-\epsilon)+\boldsymbol{B}^{2 \epsilon}\left(\frac{\Psi_{\epsilon}}{\epsilon}-\frac{\Gamma^{2}(-\epsilon)}{\epsilon}(\psi(-2 \epsilon)-\psi(1-2 \epsilon))\right), \\
I_{C 4}^{\prime \prime}= & \int \frac{d^{d} k d^{d} l}{(2 \pi)^{2 d}} \frac{(-2 \pi)^{2} \theta\left(k^{+}\right) \delta\left(k^{2}\right) e^{i(k b)_{T}} \theta\left(l^{+}\right) \delta\left(l^{2}\right) e^{i(l b)_{T}}}{\left(k^{+}+l^{+}+i \delta^{+}\right)\left(k^{-}+l^{-}+2 i \delta^{-}\right)\left((k+l)^{2}-i 0\right)}=-I_{C 1}^{\prime \prime}-B^{2 \epsilon} \frac{\Psi_{\epsilon}+\ln 2+\psi(-\epsilon)-\psi(-2 \epsilon)}{1-2 \epsilon} .
\end{aligned}
$$

The explicit form of $Q(\epsilon)$ that we found is a complicated expression that involves derivatives of the ${ }_{3} F_{2}$-hypergeometric function. The $\epsilon$ expansion of $Q(\epsilon)$ reads 


$$
\begin{aligned}
Q(\epsilon)= & -\frac{23 \pi^{4}}{1440}-\frac{\pi^{2}}{6} \ln ^{2} 2-2 \gamma_{E} \zeta_{3}+\frac{\ln ^{4} 2}{6} \\
& +4 \operatorname{Li}_{4}\left(\frac{1}{2}\right)-\frac{\ln 2}{2} \zeta_{3}+\mathcal{O}\left(\epsilon^{1}\right) .
\end{aligned}
$$

\section{APPENDIX B: GAUGE INVARIANCE AND $\delta$ REGULATOR}

It is legitimate to study whether the $\delta$ regulator that we have used interferes with the gauge invariance of the final result. Given the implementation of the regulator at the level of the operator in Eq. (5), one would naively expect that eventual gauge-breaking pieces appear as positive powers of the $\delta$ parameter and so disappear in the limit $\delta^{ \pm} \rightarrow 0$. However in the perturbative computations we observe that a naive implementation of the $\delta$ regulator breaks gauge invariance.

The problems with gauge invariance are caused by the loop integrals which do not contain any rapidity divergences and which should then not be calculated while keeping the $\delta$ 's finite. In this regard, within our NNLO calculation, only the longitudinal " $k^{\mu} k^{\nu}$ part" of the gluon self-energy $\Pi^{\mu \nu}$ is worrisome, since in some of the diagrams it can cancel the rapidity divergences and allow for the integrals to be done in pure dimensional regularization, after setting all $\delta$ 's to zero. In particular, these integrals appear only in diagrams $C, O$, and $P$. Below, we explain in detail what is the issue in diagram $C$, but similar considerations apply to $O$ and $P$.

Diagram $C$ is given by the following integral:

$$
\begin{aligned}
\mathrm{SF}_{C}= & g^{2} C_{F} \int \frac{d^{d} k d^{d} l}{(2 \pi)^{2 d}} \\
& \times \frac{-\bar{n}^{\mu} n^{\nu}}{\left(k^{+}+i \delta^{+}\right)\left(-k^{-}-i \delta^{-}\right)} \frac{-i}{k^{2}} \Pi_{R}^{\mu \nu}(k) \frac{-i}{k^{2}},
\end{aligned}
$$

where $\Pi_{R}^{\mu \nu}$ is the renormalized gluon self-energy. It reads

$$
\begin{aligned}
\Pi_{R}^{\mu \nu}(k)= & i a_{s} \frac{(-1)^{-\epsilon}}{\left(k^{2}+i 0\right)^{\epsilon}}\left(g^{\mu \nu} k^{2}-k^{\mu} k^{\nu}\right) \Gamma(\epsilon) \\
& \times \frac{4 \Gamma(1-\epsilon) \Gamma(3-\epsilon)}{\Gamma(5-2 \epsilon)}\left[C_{A}(5-3 \epsilon)-T_{f} N_{f} 4(1-\epsilon)\right] \\
& +i\left(1-Z_{3}^{\mathrm{MS}}\right)\left(g^{\mu \nu} k^{2}-k^{\mu} k^{\nu}\right),
\end{aligned}
$$

with

$$
Z_{3}^{\mathrm{MS}}=1-\frac{a_{s}}{\epsilon}\left(\frac{4}{3} T_{R} N_{f}-\frac{5}{3} C_{A}\right)+\ldots
$$

As can be seen, the longitudinal $k^{\mu} k^{\nu}$ part of the gluon selfenergy will give a term where $\delta$ 's can be set to zero at the level of the integrand, since there are no rapidity divergences to be regularized. Thus, this term can be calculated in pure dimensional regularization, giving zero. The same logic applies to diagrams $O$ and $P$, where the longitudinal part of the self-energy subdiagram will give terms that do not contain rapidity divergences and which are zero in pure dimensional regularization.

In conclusion, the gauge invariance of the final result is completely guaranteed as far as the $\delta$ regulator is implemented consistently, i.e. respecting the axiomatic properties of dimensional regularization. In other words, $\delta$ 's should be set to zero in every integral that does not contain rapidity divergences.
[1] J. C. Collins, Foundations of Perturbative QCD (Cambridge University Press, Cambridge, England, 2011).

[2] M. G. Echevarria, A. Idilbi, and I. Scimemi, J. High Energy Phys. 07 (2012) 002.

[3] M. G. Echevarria, A. Idilbi, and I. Scimemi, Phys. Lett. B 726, 795 (2013).

[4] M. G. Echevarria, A. Idilbi, and I. Scimemi, Phys. Rev. D 90, 014003 (2014).

[5] S. M. Aybat and T. C. Rogers, Phys. Rev. D 83, 114042 (2011).

[6] A. Bacchetta and A. Prokudin, Nucl. Phys. B875, 536 (2013).

[7] A. V. Belitsky, X. Ji, and F. Yuan, Nucl. Phys. B656, 165 (2003).

[8] A. Idilbi and I. Scimemi, Phys. Lett. B 695, 463 (2011).

[9] M. Garcia-Echevarria, A. Idilbi, and I. Scimemi, Phys. Rev. D 84, 011502 (2011).
[10] M. G. Echevarria, I. Scimemi, and A. Vladimirov, Phys. Rev. D 93, 011502 (2016).

[11] M. G. Echevarria, I. Scimemi, and A. Vladimirov (to be published).

[12] A. V. Belitsky, Phys. Lett. B 442, 307 (1998).

[13] J. C. Collins and A. Metz, Phys. Rev. Lett. 93, 252001 (2004).

[14] X.d. Ji, J.-P. Ma, and F. Yuan, Phys. Rev. D 71, 034005 (2005).

[15] X. d. Ji, J.-P. Ma, and F. Yuan, Phys. Lett. B 597, 299 (2004).

[16] Y. Li, S. Mantry, and F. Petriello, Phys. Rev. D 84, 094014 (2011).

[17] J. Y. Chiu, A. Jain, D. Neill, and I. Z. Rothstein, J. High Energy Phys. 05 (2012) 084.

[18] T. Becher and M. Neubert, Eur. Phys. J. C 71, 1665 (2011). 
[19] T. Gehrmann, T. Lubbert, and L. L. Yang, Phys. Rev. Lett. 109, 242003 (2012).

[20] T. Gehrmann, T. Luebbert, and L. L. Yang, J. High Energy Phys. 06 (2014) 155.

[21] S. Catani, L. Cieri, D. de Florian, G. Ferrera, and M. Grazzini, Eur. Phys. J. C 72, 2195 (2012).

[22] A. A. Vladimirov, J. High Energy Phys. 08 (2014) 089.

[23] I. O. Cherednikov and N. G. Stefanis, Nucl. Phys. B802, 146 (2008).

[24] J. G. M. Gatheral, Phys. Lett. 133B, 90 (1983).

[25] J. Frenkel and J. C. Taylor, Nucl. Phys. B246, 231 (1984).

[26] G. F. Sterman, AIP Conf. Proc. 74, 22 (1981).
[27] A. A. Vladimirov, Phys. Rev. D 90, 066007 (2014).

[28] A. A. Vladimirov, J. High Energy Phys. 06 (2015) 120.

[29] V. S. Dotsenko and S. N. Vergeles, Nucl. Phys. B169, 527 (1980).

[30] R. A. Brandt, F. Neri, and M. a. Sato, Phys. Rev. D 24, 879 (1981).

[31] M. G. Echevarria, A. Idilbi, and I. Scimemi, Int. J. Mod. Phys. Conf. Ser. 25, 1460005 (2014).

[32] M. G. Echevarria, T. Kasemets, P. J. Mulders, and C. Pisano, J. High Energy Phys. 07 (2015) 158.

[33] M. G. Echevarria, A. Idilbi, A. Schäfer, and I. Scimemi, Eur. Phys. J. C 73, 2636 (2013). 